\title{
El encierro carcelario. Impacto en las emociones y los cuerpos de las mujeres presas
}

\section{Imprisonment. Impact in the emotions and bodies of incarcerated women}

\author{
Estíbaliz DE Miguel CALvo \\ Universidad de Deusto \\ estibaliz.miguel@deusto.es
}

Recibido: 03/02/2014

Revisado: $12 / 02 / 2014$

Aceptado: 31/07/2014

Disponible on line: 10/12/2014

\section{Resumen}

A partir del estudio empírico realizado en el módulo de mujeres de una cárcel vasca, en el artículo se argumenta que la experiencia del encarcelamiento tiene un impacto en las emociones y los cuerpos de las mujeres presas que se trasluce en un deterioro de su salud mental, y en la vivencia de una sexualidad mediada por la institución penitenciaria. Respecto a la salud, se constata un empeoramiento del estado general, especialmente en lo que se refiere a la salud mental o al estado psicoemocional. Las entrevistadas manifiestan haber padecido ataques de ansiedad, depresión y, en algunos casos, intentos de suicidio. La respuesta del personal sanitario de la prisión es una prescripción excesiva de medicamentos, a la que algunas presas se oponen de manera explícita. En lo que respecta a la vivencia de su sexualidad, nos centramos en los encuentros sexuales en la cárcel (llamados vis a vis íntimos), que las participantes definen como negativos en la medida en que tienen lugar en un espacio carcelario, en un día y hora fijadas por la institución, y bajo la mirada del personal de la prisión.

Palabras clave: cárceles, estudios feministas, emoción, salud, sexualidad.

\begin{abstract}
Drawing on the empirical research conducted within a women's unit in a Basque prison, the article argues that the experience of incarceration has an impact on the emotions and bodies of women in prison, which is made visible in the deterioration of the mental health and the experience of sexuality mediated by the penitentiary institution. Regarding their health, the general status gets worse, especially in respect of their mental health or psych emotional situation. Interviewees expressed having suffered from nervous breakdowns, depression and suicide attempts in some cases. The response of the medical staff of the prison is excessive prescription of drugs, to which some dams oppose explicitly. Regarding the experience of sexuality, we focus on sexual encounters in prison (called Vis a Vis intimate), defined by the participants as negative as they take place in a prison space, in a day and time set by the institution, and under the gaze of prison staff.
\end{abstract}

Keywords: penal institutions, gender studies, emotion, health, sexuality.

Referencia normalizada: De Miguel Calvo, E. (2014): «El encierro carcelario. Impacto en las emociones y los cuerpos de las mujeres presas». Cuadernos de Trabajo Social, 27(2): 395-404.

Sumario: Introducción. 1. Investigación sobre las mujeres presas en la cárcel de Nanclares de Oca. 2. Impacto del encarcelamiento en la salud. 3. Impacto del encarcelamiento la sexualidad de las mujeres. Los vis-à-vis íntimos. 4. Conclusión. 5. Referencias bibliográficas.

\section{Introducción ${ }^{1}$}

El encarcelamiento de mujeres es uno de los campos menos desarrollados en ciencias sociales. La literatura científica en el Estado Español es muy reciente y fragmentaria y no ha sido hasta los últimos quince años en que los trabajos relacionados con las mujeres en prisión se han empezado a desarrollar.

${ }^{1}$ La investigación que se presenta ha sido financiada por el Departamento de Educación, Universidades e Investigación del Gobierno Vasco, a través de una beca FPI-Formación de Personal Investigador (2008-2011). 
Las principales reflexiones se han inclinado hacia la relación entre la baja proporción de mujeres en el sistema penitenciario y las carencias materiales y de políticas específicas de género para las mujeres encarceladas (Almeda, 2002, 2003, 2005, 2006; Cruells e Igareda, 2005). Algunos autores y autoras, por su parte, se muestran preocupadas por las consecuencias del encarcelamiento de los niños y niñas (Jabardo, 1993; Jiménez y Palacios, 1997; Yagüe Olmos, 2007) o del rol de las mujeres presas como madres y cuidadoras (Igareda, 2009). Criminólogas feministas del mundo anglosajón (Smart, 1977; Carlen, 1983, 1998; Carlen y Worrall, 1987; Worrall, 1990) han sido fuente de inspiración para las investigaciones de nuestro contexto (citados en Almeda, 2003, pp.15-17).

Las consecuencias del encarcelamiento para las mujeres han tendido a analizarse desde el punto de vista del fuerte impacto que la prisión conlleva para su entorno familiar y el consiguiente malestar que supone para las mujeres presas el no poder desempeñar su papel de madres y cuidadoras. Sin embargo, muchos otros elementos entran en juego a la hora de comprender la experiencia del encarcelamiento de las mujeres en toda su complejidad, no referido exclusivamente a su papel como sostenedoras del hogar y seres-para-otros (Lagarde, 2005).

Aquí, el foco se sitúa en dimensiones como la subjetividad y el cuerpo, la propia salud y las emociones, aspectos que ya han sido descritos en otros trabajos sobre encarcelamiento pero que no han llegado a constituir una reflexión autónoma situada en el contexto de la sociología de las emociones en la búsqueda por articular las relaciones entre contexto, mente y cuerpo (Freund 1990). Más allá del enfoque bio-médico de la salud, que a menudo adolece de una visión excesivamente centrada en el cuerpo, se busca relacionar la materialidad con un enfoque sociocultural, que dé cuenta de las condiciones sociales y culturales que dan lugar a los malestares. Se trata también de buscar de qué manera las emociones se encarnan corporalmente (Lupton, 1998) para trazar puentes entre la sociología de las emociones y la sociología de la salud y la enfermedad, que puedan dar lugar a una comprensión holística acerca de las mujeres presas que permita captar cómo la intersección de ejes de estratificación social (clase social, género y encarcelamiento entre otras) se «in-corpora» en la propia subjetividad y el propio cuerpo de las protagonistas.

\section{Investigación sobre las mujeres presas en la cárcel de Nanclares de Oca}

El presente estudio se basa en las 49 entrevistas semiestructuradas realizadas a mujeres de la prisión de Nanclares de Oca (Álava, Comunidad Autónoma de Euskadi) y en la observación participante desarrollada a lo largo de 14 meses $^{2}$. Durante ese tiempo, quien escribe asistió semanalmente a diferentes actividades y espacios de los dos módulos del departamento de mujeres de la prisión. Esto me permitió conocer diferentes aspectos de la vida cotidiana del encierro de las mujeres, que fueron registrados en el diario de campo. La observación participante tenía como objetivos explorar las condiciones de vida de las mujeres en prisión y la importancia de las relaciones amorosas e íntimas en el ambiente carcelario. Al mismo tiempo, la «estancia con las mujeres» presas (Lagarde, 2005) dio la posibilidad de ganar su confianza para que participaran en la investigación. La autorización por parte de la Dirección General de Instituciones Penitenciarias permitía, además, usar la grabadora durante las entrevistas, lo cual suponía toda una novedad con respecto a los estudios desarrollados previamente, que tradicionalmente habían tenido que salvar numerosos obstáculos que la institución penitenciaria ponía a quienes querían investigar esta materia ${ }^{3}$.

El recinto carcelario de Nanclares de Oca se abrió en 1982 y hasta 2012 constituyó la única prisión de cumplimiento de condena de las tres erigidas en la Comunidad Autónoma de Euskadi. Con 470 celdas habitables, oscilaba entre 700 y 800 personas presas, de las cuales entre $60 \mathrm{y}$ 70 eran mujeres, localizadas en un departamento que contaba con dos módulos (Salhaketa, 2005). Actualmente, la cárcel de Nanclares de Oca ha sido sustituida por un nuevo complejo carcelario, el Centro Penitenciario Álava/Araba.

2 Entre noviembre de 2007 y diciembre de 2008.

3 Desde Ríos Martín y Cabrera Cabrera, (1998, 2002) hasta Alvarado Sánchez (2012) la generalidad de los estudios realizados en el Estado español mantiene que la institución penitenciaria se ha mostrado poco colaboradora y recelosa ante la entrada de personas investigadoras. 
El método de muestreo fue de autoselección, es decir, todas las mujeres contactadas en prisión fueron informadas sobre el sentido de la presencia de la investigadora en el departamento $\mathrm{y}$ fueron invitadas a participar mediante una entrevista que se realizaría en privado dentro de la cárcel. La muestra no es estadísticamente representativa dado su marcado carácter cualitativo, pero se acerca a las descripciones sociodemográficas de la literatura científica referida a las mujeres en prisión, en lo referente a la alta proporción de mujeres gitanas (Barañí, 2001) y extranjeras (Martin Palomo, Miranda López y Vega Solís, 2005; Ribas, Almeda y Bodelón, 2005), pero también a la importante presencia de (ex)consumidoras de drogas (Gañan y Gordon, 2000) y madres. También se buscó diversidad en la edad de las participantes.

Así, la muestra está compuesta por mujeres entre 20 y 64 años, resultando una media de edad de 35,7 años. Prácticamente la mitad de las mujeres entrevistadas habían nacido en la Comunidad Autónoma de Euskadi, mientras que una cuarta parte eran originarias de otras partes del Estado español. Además, una de cada tres había nacido en un país extranjero. $\mathrm{La}$ mitad de ellas eran toxicómanas o habían abusado de las drogas en algún momento de su vida. Preguntadas sobre su etnia, casi tres cuartas partes se identificaron como «payas» (no gitanas) mientras que el resto se identificaron como gitanas. Por otro lado, dos terceras partes eran madres y tres cuartas partes tenían una relación de pareja en el momento de la entrevista, entre ellas, cerca de la mitad era con un hombre que se encontraba en prisión en ese momento, bien sea en la misma prisión de Nanclares o en alguna otra.

La entrevista semiestructurada elaboraba información acerca de los perfiles e itinerarios sociales, familiares y personales de las participantes tales como: familia de origen, residencia e ingresos, familia actual, formación y situación laboral, situación penal y penitenciaria, estado de salud, violencia y malos tratos, impacto del encarcelamiento y perspectivas de futuro. Todo ello en el marco del estudio de las «Relaciones amorosas de pareja en las trayectorias vitales de las mujeres encarceladas» que elaboraba principalmente información relativa a las relaciones de pareja, tanto en el momento de la investigación como en el tiempo antes de prisión.

\section{Impacto del encarcelamiento en la salud}

\subsection{La prisión como entorno agresivo}

Para comprender el impacto del encarcelamiento es necesario tener en cuenta la vivencia negativa de las relaciones dentro de prisión y caer en la cuenta que la precarización de la salud tiene mucho que ver con la corporalización de esos malestares. El sistema penitenciario es un sistema social alternativo, donde están muy acentuadas las relaciones de poder y las dinámicas de género. El asfixiante ambiente de la prisión, tanto en su vertiente arquitectónica como en su configuración social, es fuente primordial de padecimientos psicológicos y emocionales de las mujeres presas.

La pena privativa de libertad, a pesar de que en sus fundamentos legales tiene como principal objetivo la resocialización de las personas presas, en la práctica está principalmente orientada a la guarda y custodia, tanto en lo que respecta a la organización general de la vida en prisión, como en la arquitectura de los centros penitenciarios y las políticas penitenciarias. El propio personal sanitario reconoce esta priorización de lo regimental sobre lo asistencial entre las carencias estructurales de los centros penitenciarios españoles (CAPRI, 2003).

La vida en prisión se convierte en un sistema social alternativo con su propia cultura, sus normas y hábitos, y sus propias dinámicas de relación, tanto entre las personas presas y el funcionariado, como entre las personas presas constituyendo una «institución total» (Goffman, 1998). Uno de los rasgos más relevantes de la vida cotidiana tras los barrotes es la falta de intimidad de las personas encarceladas, quienes en la mayoría de las ocasiones tienen que compartir celda con otras personas que no formaban parte de su red de relaciones antes del encierro. El resto de dependencias de la prisión (salas, galerías, patio, etc.) tampoco permiten encontrar momentos de intimidad, menos aún de silencio o cierta tranquilidad.

El propio encierro, el hecho de estar tras los muros de una prisión y encerrada en una celda produce sensación de enclaustramiento. Rosa habla de lo duro que se le hace el tiempo de después de comer, cuanto tienen que permanecer encerradas en las celdas:

De 2 a 5 (de la tarde), cuando nos chapan. Lo llevo muy mal, lo llevo fatal. Por la noche, vaya... 
como luego que si ves la tele o que si lees un rato, tal... te echas ya a dormir y bueno. Pero los medios días que yo no duermo, estar ahí cerrada, puff (resopla) se me hace cuesta arriba, ya lo saben ellas (las funcionarias), a mí se me hace cuesta arriba, pero no queda otra.

A ello hay que añadir que el día a día de la prisión está marcado fuertemente por las relaciones de poder, ya sea en las relaciones funcionaria-presa, como en las relaciones presa-presa. De hecho, las participantes en el estudio manifestaban que no se podía confiar en otras internas, ya que era habitual que desvelaran confidencias que habían sido contadas, incluso que usaran cierta información para dañar a otras compañeras. Si bien la observación participante encontró que esta agresividad mutua convivía con importantes gestos de solidaridad, es importante reconocer el sentimiento de amenaza que las entrevistadas percibían en la relación con otras internas. Las mujeres entrevistadas tendían a describir el ambiente de relación entre las presas como hostil y de desconfianza, hasta el punto de que algunas de ellas manifestaron que lo peor de la prisión no era la institución, sino la dureza de las condiciones de vida en prisión, precisamente debido a la hostilidad de relaciones entre presas.

Esto es como un «Gran Hermano» pero a lo grande, a lo grande porque todos los días viendo las mismas caras somos siempre las mismas. $\mathrm{Si}$ viene gente nueva, tienes que andar observando para ver si te va a meter una puñalada trapera, si puedes ser su amiga. Siempre tienes que estar mirando p'atrás. Dices ¿cómo será la cárcel? Un suplicio, no es un castigo es un suplicio.

Por lo tanto, la cotidianidad del encierro puede describirse como asfixiante, arquitectónicamente hablando, y amenazante en lo referente a las relaciones, lo cual conducen a un estado de permanente ansiedad (Valverde Molina 1991).

\subsection{La salud de las mujeres encarceladas}

Para comprender el estado de salud de las mujeres encarceladas, es importante tener en cuenta el precario estado de salud de muchas de ellas antes de entrar en prisión, debido a las condiciones de desigualdad social previas al encarcelamiento, a las experiencias personales traumáticas (abusos sexuales, violencia de género o problemas familiares), las enfermedades o los trastornos mentales y conductas de riesgo en uso de drogas o conductas sexuales. Aparece, pues, una fuerte relación entre las desigualdades sociales y las desigualdades en salud, con la variable de género como eje vertebrador principal. De hecho, varios de los informes europeos del MIP apuntan que «las necesidades de salud de las mujeres presas, tanto físicas como psicológicas son distintas [a las de los hombres] y estas necesidades sanitarias no son atendidas de forma adecuada» (Cruells y Igareda, 2005, p. 88). En esta línea, las mujeres participantes en la investigación manifestaron problemas de salud antes de entrar en prisión en porcentajes nada desdeñables. Así, más de una de cada diez afirmaron tener una o más de las siguientes dolencias antes de su ingreso: enfermedad crónica, problemas psíquicos, alcoholismo, VIH habitualmente relacionado con el consumo de drogas $\mathrm{u}$ otros problemas de salud. Cerca de la mitad de ellas era o habían sido toxicómanas.

Una vez en prisión, el estado de salud tiende a deteriorarse tanto física como psicológicamente. El ESDIP (Dirección General del Plan Nacional sobre Drogas, 2011) recogió que el 67 por ciento de la población penitenciaria manifestó que su salud había empeorado desde su entrada en prisión. Respecto a la investigación que aquí se presenta, preguntadas si durante el tiempo en que se encontraban en prisión habían padecido o padecían algún problema de salud, una de cada cinco refiere a problemas psíquicos y más de una de cada tres participantes en la investigación refieren problemas de salud varios, generalmente relacionados con el impacto físico-emocional del encierro, como describiremos más adelante. La mitad, estaban recibiendo tratamiento en el momento de la entrevista, de las cuales casi la mitad tomaban medicación para síntomas de depresión o ansiedad, dificultad para conciliar el sueño o similares alteraciones psicológicas, por lo general derivadas del padecimiento del encierro en prisión.

Por su parte, y de manera similar, el estudio de Manzanos y Balmaseda (2003) sobre la situación de las mujeres en las cárceles del País Vasco recoge que las mujeres presas perciben graves problemas de salud en sí mismas y sus compañeras o situaciones que exigen un tratamiento médico especializado. Más de una de cada cuatro mujeres participantes expresaron que 
padecían una enfermedad larga o crónica y en casi la totalidad de los casos expresaron que en la cárcel existían problemas psíquicos, padecimientos derivados de conductas de riesgo en ámbitos como el consumo de drogas (hepatitis y SIDA) y problemas de salud relacionados con la salud sexual y reproductiva (enfermedades de transmisión sexual y problemas derivados de la gestación). Respecto a los problemas padecidos los seis últimos meses, reportan dolencias que son consecuencia de las condiciones de encierro, como dolores de cabeza en casi todos los casos, pérdida de visión en la mitad de ellas y también para una de cada dos molestias tales como pérdida de audición, vómitos y fiebre.

\subsection{Estado de salud mental en prisión}

Analizamos ahora las respuestas que dieron las entrevistadas de nuestra investigación ante la pregunta de si habían tenido algún tipo de padecimiento psicológico durante su tiempo en prisión, de una lista dada. El fuerte impacto de la experiencia de encarcelamiento en las mujeres es notorio si tenemos en cuenta que más de la mitad de ellas dijeron que la cárcel les había traído síntomas de depresión, y/o agobio y claustrofobia. Además, tres cuartas partes de las participantes reportó síntomas de ansiedad y más de la mitad haber sufrido algún ataque de ansiedad durante su estancia en prisión. En menor medida, pero aún de manera muy importante, más de una de cada tres manifestaban que la situación de encierro carcelario había traído consigo deseos de venganza y en un porcentaje similar, agresividad. Asimismo, una de cada tres afirmó que la cárcel le había producido desequilibrios mentales y una de cada diez manifestó haber intentado suicidarse en algún momento de su tiempo de encarcelamiento.

Estos resultados están en clara consonancia con las cifras manejadas por otros estudios similares. En el mencionado trabajo de Manzanos \& Balmaseda (2003) la totalidad de las mujeres participantes afirmaron que la prisión les había causado desequilibrios psicológicos y somáticos de diversa gravedad como sensación de agobio y claustrofobia, aislamiento de la familia, depresión y ansiedad contenida.

Investigaciones con la población penitenciaria masculina también revelan la importante presencia de problemas de salud mental. Por un lado, el estudio de Instituciones Penitenciarias
(2006) sobre salud mental en el medio penitenciario que analiza historias clínicas de hombres encarcelados, apunta antecedentes psiquiátricos en el 17,6 por ciento de los presos y antecedentes de abuso de drogas en un 42,2 por ciento. Ya en prisión, uno de cada cuatro presenta uno o dos diagnósticos psiquiátricos, sin contar las drogodependencias, y si se incluyen estas hablamos de casi la mitad de la población. El tratamiento psiquiátrico más habitual es el farmacológico (antidepresivos, principalmente). Se comprueba, por tanto, un elevado porcentaje de antecedentes de trastornos mentales previos al ingreso en prisión y una más elevada tasa aún una vez encarcelados.

Por otro lado, el Grupo Preca (2011) en el estudio más amplio llevado a cabo hasta el momento en España en lo que se refiere la prevalencia de enfermedad mental entre la población penitenciaria, encontró en una muestra de varones que la prevalencia era 5 veces superior a la población general, con una alta relación entre enfermedad mental y consumo de drogas. Concretamente, la prevalencia de trastorno mental es del 84,4 por ciento, siendo el consumo de sustancias el más frecuente (76,2 por ciento), seguido de trastorno de ansiedad ( 45,3 por ciento), trastorno afectivo (41 por ciento) y trastorno psicótico en un 10 por ciento de los casos.

No obstante, existen indicios para pensar que la salud mental de las mujeres en las prisiones está más deteriorada que en el caso de los hombres. El proyecto MIP (Cruells e Igareda, 2005) apunta el mayor sufrimiento psicológico de las mujeres (que los hombres) en el interior de los centros penitenciarios. Su informe de Inglaterra y Gales pone de manifiesto que las mujeres encarceladas cometen más suicidios y autolesiones que los hombres, mientras que en la población general ellos se suicidan dos veces más que ellas.

\subsection{La respuesta medicalizadora del siste- ma penitenciario}

El estado de salud general de la población penitenciaria en general y de las mujeres presas en concreto, por tanto, es negativo. A los trastornos físicos y psicológicos derivados de las condiciones de vida previas al encarcelamiento, se le suman los trastornos derivados de condiciones de vida en prisión. Tampoco se deben olvidar las graves deficiencias de atención sanitaria en los 
centros penitenciarios: excesiva medicalización, falta de abordaje global, escasez de tratamiento psicológico, deficiencias de atención ginecológica y en anticoncepción (Manzanos y Balmaseda, 2003; Cruells e Igareda, 2005).

En la misma línea se dirigen las críticas de los propios profesionales sanitarios penitenciarios, cuando señalan que la priorización de lo regimental frente a lo asistencial, la escasez y motivación de los profesionales así como la descoordinación con la red asistencial extra-penitenciaria son carencias estructurales importantes que conllevan, entre otras cosas, la falta de implicación de algunos profesionales en su trabajo (Preca, 2011).

La cuestión de la medicación, pues, resulta problemática en este contexto, tal y como manifestaron varias de las mujeres entrevistadas:

Aquí todo lo solucionan con pastillas. Los médicos. Bah, si estás nerviosa, una pastilla. Pero que no, yo lo que necesito es hablar, no necesito pastillas. ¿Prepotente? No soy prepotente. A mí me gusta que me escuchen.

María pone de manifiesto una sobre-medicación, de la que se quejaban también otras presas, y expresa una demanda de escucha. Es probable que gran parte del desasosiego que las mujeres encarceladas experimentan se viera aliviado si pudiesen contar con personas que les dedicasen el tiempo suficiente como para escuchar sus cuitas. Al menos, ese es el clamor de la entrevistada.

Pero ¿a qué puede deberse este abusivo recurso de los psicofármacos para la población penitenciaria femenina? Analicemos las tres causas que apuntan Cruells y Torrens (2004): (1) la escasez de personal, (2) el uso de la medicación como mecanismo de control y, (3) los estereotipos de género por los cuales las mujeres son por naturaleza más propensas a enfermedades mentales, más histéricas, sensibles y depresivas.

En cuanto a la falta de personal, siendo el hacinamiento un problema endémico en el sistema penitenciario español (Cabrera, 2011; Aranda y Rivera, 2012), no es difícil imaginarse que el personal penitenciario encargado de la asistencia médica, se encuentre desbordado y que habitualmente recurra a la solución que parece más fácil y rápida: recetar medicamentos. Este tipo de respuesta médica se encuentra también fuera de los muros de la prisión: la respuesta medicalizadora está más orientada a eliminar el síntoma que a tratar la raíz del problema de salud (Sanfélix-Gimeno, Peiró y Meneu, 2012).

Pero el problema de la excesiva prescripción de medicación para padecimientos psicosomáticos puede tener una lectura más compleja y se puede comprender en la confluencia de diversos factores criminológicos relacionados con las dinámicas del sistema penitenciario, y también de manera más general, con la tendencia a psicopatologizar los padecimientos de las mujeres.

Además de las ideas estereotipadas heredadas de los «padres» de la antropología criminal acerca de las mujeres delincuentes, ya descritas por Almeda (2003), esta cuestión de la excesiva medicalización de la población penitenciaria femenina se ve alimentada por la concepción estereotipada acerca de las mujeres en general quienes, no encajando en el modelo de racionalidad por definición masculina, habitualmente son catalogadas como histéricas, emocionales y enfermas mentales (Lagarde, 2005). No sólo la antropología criminal, sino también la psicología, han jugado un importante papel a este respecto (San Martín, 2007, p. 174). Un enfoque excesivamente individualizante y ciego a los condicionantes de género, fácilmente convierte en patología lo que puede ser un malestar embebido en condiciones ambientales y estructurales adversas, que puede ser comprendido desde la perspectiva de la estratificación social de las emociones.

Pasamos en el siguiente apartado al impacto del encarcelamiento en un aspecto de experiencia corporal de las mujeres en prisión: los encuentros sexuales.

\section{Impacto del encarcelamiento la sexuali- dad de las mujeres. Los vis-à-vis íntimos}

Las comunicaciones íntimas son una de las modalidades de contacto presencial con personas del exterior, entre las que se encuentran también la comunicación en locutorio o «por cristales», que es de una media hora cada semana; y las comunicaciones vis a vis, que se dividen entre familiares e intimas, de hora y media de duración. Los encuentros íntimos también se producen con personas presas, siempre que cumpla los requisitos generales de haber sido acreditada como pareja legal o de hecho, y se encuentre en la misma prisión (OSPDH, 2006, p. 30). 
Entre las mujeres entrevistadas en la cárcel de Nanclares de Oca, la mitad mantenían en ese momento o habían mantenido en otras etapas comunicaciones íntimas. Preguntadas por la opinión que les merecían las circunstancias en que se producen esos encuentros íntimos, cerca de la mitad de ellas dijeron que las circunstancias eran malas. Las críticas se dirigieron principalmente a las características de los encuentros y la falta de tiempo.

Las comunicaciones íntimas son definidas por las mujeres entrevistadas como encuentros «fríos», más por las características de la situación que por la temperatura de las salas. La prisión se vive como un espacio inadecuado para la experiencia de las relaciones afectivas y sexuales:

Fríos, fríos, son fríos (...) No me dan ganas de hacer el amor, me dan ganas de abrazar y de sentir el calor y conversar con él y cuando ya llega la hora, ya está, perdido. Son frías.

La incomodidad de los encuentros se refiere especialmente al tiempo limitado, al lugar y a la falta de intimidad e intrusión por parte del personal funcionario, llegando a producirse situaciones humillantes. Las personas presas ven cómo su intimidad y su sexualidad se ven expuestas a la vigilancia y la intromisión de la institución penitenciaria. De hecho, más de una de ellas reporta que el funcionario o funcionaria al cargo en ese momento ha entrado en la sala sin previo aviso. Tanto es así, que algunas mujeres describen a los vis a vis íntimos como una escena de prostíbulo más que propia de relaciones intimas de pareja:

Solo te falta que te dejen 5 euros en la mesilla. Una hora y media, y viene un funcionario y la abre, te tienes que vestir.

Las condiciones bien, el tiempo no porque estás tol día mirando, cronometrando, vamos, que esto parece que estás en un puticlú, es verdad, estas así... una y media, en punto (mirando el reloj). Si no, les da igual, que te pillen en bragas, a cuatro patas o encima de un armario, vamos que ni las putas hacen eso, hija que tienen más, ponen ellas el tiempo, es verdad. Vamos... como ganao.

La falta de tiempo es lo más destacado entre las participantes que respondieron acerca de las relaciones íntimas. Esta escasez de tiempo no deja opción a compatibilizar el encuentro sexual con el diálogo:

Hora y media. Controlando el jodido reloj. $\mathrm{Si}$ hablas, no haces. Y si haces, no hablas. Es que es así, en hora y media mucho no da tiempo a hacer, y hablar menos.

Precisamente, el estudio realizado por el OSPDH (2006) recogió impresiones similares por parte de las mujeres que tenían algún tipo de relación con un hombre preso. Las valoraciones de las mujeres que acuden desde el exterior de la prisión a tener un encuentro íntimo son muy similares a las de las mujeres encarceladas. Expresan que no son encuentros gratificantes por la falta de tiempo, al temporalización estricta, la no libre elección del momento del contacto íntimo y la falta de intimidad derivada del conocimiento que los demás tienen sobre el contenido sexual de la visita (2006, p. 32). Las participantes manifestaron que resulta público y notorio para las personas involucradas en la maquinaria penitenciaria que la mujer acude con el fin de tener un encuentro íntimo de connotaciones sexuales con un hombre encarcelado, produciéndose situaciones humillantes para ellas.

Consecuentemente, y de manera paradójica, no puede decirse que durante las comunicaciones denominadas «íntimas» se preserve la intimidad ni se den las condiciones necesarias para un encuentro afectivo y sexual. Y en esto las mujeres, ya sean presas o parejas de presos, salen peor paradas ya que ven sometidos sus cuerpos a la vigilancia, la regulación y el control de un sistema sexista. El momento y el espacio de la «intimidad» y la sexualidad en prisión, es expresión máxima del gran impacto que el encarcelamiento tiene para la vida amorosa, para los cuerpos y la sexualidad de las mujeres. En la escenificación de la comunicación íntima se hace escarnio público y mofa del encuentro. Esa publicidad y esa falta total de la posibilidad de hacer ese espacio algo propio lo que la sitúa en un control menor del escenario sexual que la prostituta.

\section{Conclusión}

En muy contadas ocasiones la situación de las mujeres en prisión es objeto de interés en las ciencias sociales, más allá del que suscita en el ámbito jurídico o criminológico. Para la socio- 
logía, las cuestiones relacionadas con un puñado de mujeres, un porcentaje residual de la población penitenciaria, no ha despertado especial interés. Sin embargo, la cárcel es un «espejo» de aumento de las dinámicas sociopolíticas, y en el caso que nos ocupa, una interesante caja de resonancia de las dinámicas sociales de género, condiciones estructurales que se encarnan en los cuerpos y la subjetividad de las mujeres presas.

El fuerte impacto en la salud mental del encarcelamiento y la respuesta medicalizadora pone de manifiesto las carencias sanitarias del sistema penitenciario y saca a la luz las carencias en la atención de las dolencias de las mujeres. La humillación de que son objeto las mujeres en los encuentros sexuales con sus parejas dentro de prisión, son una muestra de la objetualización de los cuerpos de las mujeres, que se muestra con toda su crudeza tras los muros de las cárceles.

Finalmente, ante el elevado coste personal y social del encarcelamiento de mujeres pueden proponerse numerosas medidas que puedan paliar tal sufrimiento mientras se encuentran en prisión. Sin embargo, la drástica reducción de los niveles de encarcelamiento de mujeres pobres parece ser la medida más efectiva, tal y como proponen la mayoría de los trabajos dedicados a esta temática y los informes de agencias internacionales.
Así, Almeda (2002, pp. 207-213) propone iniciar un proceso de despenalización y descriminalización de determinadas conductas tipificadas por el Código Penal que no causan daño directo contra las personas, concretamente, los delitos contra la salud pública, lo cuales están altamente penalizados en nuestro sistema. Paralelamente, propone articular medidas alternativas a la ejecución de las penas o sustitutivas de ellas. Naciones Unidas, por su parte, se hace eco de los informes de personas expertas y «alienta a los Estados Miembros a aprobar legislación para establecer medidas sustitutivas del encarcelamiento y dar prioridad a la financiación de esos sistemas, así como a la elaboración de los mecanismos necesarios para su aplicación» (Reglas de Bangkok ONU, 2011, Regla 5)

Este panorama, por tanto, clama por la introducción de cambios en el sistema de servicios sociales y penitenciarios, que logren la mejora en la calidad de vida de las mujeres una vez excarceladas. Y es una llamada a los servicios sociales y al mundo asociativo, para que tomen conciencia de la precaria situación en que se encuentran las mujeres presas, la cual requiere una respuesta articulada desde los grupos de asistencia social y apoyo a mujeres (Almeda, 2002, 2003).

\section{Referencias bibliográficas}

Almeda, E. (2002). Corregir y Castigar: El ayer y hoy de las cárceles de mujeres. Barcelona: Bellaterra.

Almeda, E. (2003). Mujeres Encarceladas. Barcelona: Ariel.

Almeda, E. (2005). Women's Imprisonment in Spain. Punishment and Society, 7, 183-199.

Almeda, E. (2006). Mujeres y Cárceles: pasado y presente de las cárceles femeninas en España. Comunicación. Congreso Penitenciario Internacional. La Función Social de La Política Penitenciaria C1. Barcelona.

Alvarado, R.(2011). Perspectiva histórica y problemas actuales de la institución penitenciaria en España. Las mujeres encarceladas toman la palabra. Madrid: UCM.

Aranda, M. y Rivera, I. (2012). Problemáticas detectadas en el sistema penitenciario español (y algunas propuestas). Critica Penal y Poder, 3, 121-131.

Barañi, E. (2001). Mujeres gitanas y sistema penal. Madrid: Metyel.

Cabrera, P. J. (2011). Exclusión social y prisión. Documentación Social, 161, 29-41.

Carlen, P. (1983). Women's imprisonment: a study in social control. Londres: Routledge and Kegan Paul.

Carlen, P. (1998). Sledgehammer. Women's imprisonment at the Millenium. Londres: Macmillan Press.

Carlen, P. y Worrall, A. (1987). Gender, Crime and Justice. Milton Keynes: Open University Press.

CAPRI. (2003). Informe CAPRI sobre la calidad de la asistencia sanitaria en centros penitenciarios españoles. Revista Española de Sanidad Penitenciaria, 5 (2), 38-48. Recuperado de: http://www.sanipe.es/OJS/index.php/RESP/article/view/243/536. 
Cruells, M. y Torrens M. (2004).Mujeres, integración y prisión. Un análisis de los procesos de integración sociolaboral de las mujeres presas en Europa. Recuperado de: www.surt.org/mip/docs/ Borrador-\%20ES\%20final.doc.

Cruells, M. e Igareda, N. (eds.). (2005). Mujeres, integración y prisión. Barcelona: Aurea.

Dirección General del Plan Nacional sobre Drogas. (2011). Encuesta sobre Salud y Consumo de Drogas en internados en instituciones penitenciarias (ESDIP) Recuperado de: http://www. pnsd.msc.es/Categoria2/observa/pdf/ESDIP_2011.pdf.

Freund, P. (1990).The Expressive Body: A Commond Ground for the Sociology of Emotions and Health and Illness. Sociology of Health and Illness 12 (4), 452-77.

Goffman, E. (1998). Internados. Ensayos sobre la situación social de los enfermos mentales. $6^{\text {a }}$ ed. Buenos Aires: Amorrortu.

Igareda, N. (2009). La maternidad de las mujeres presas. En: Género y dominación. Críticas feministas del derecho y del poder. Barcelona: Anthropos.

Instituciones Penitenciarias. (2006). Estudio sobre salud mental en el medio penitenciario. Recuperado de: http://www.msssi.gob.es/organizacion/sns/planCalidadSNS/boletinAgencia/boletin10/ estudi_saludMental_medio_penitenciario.pdf.pdf.

Jabardo, M. (1993). La mujer y sus hijos en prisión. Eguzkilore, 7, 93-106.

Jiménez, J. y Palacios, J. (1997). Niños y madres en prisión. Desarrollo Psicosociobiológico de los niños residentes en los centros penitenciarios. Madrid: Ministerio de Trabajo y Asuntos Sociales.

Lagarde, M. (2005). Los cautiverios de las mujeres: madresposas, monjas, putas, presas y locas. $4^{\mathrm{a}}$ ed. México: UNAM.

Lupton, D. (1998). The Emotional Self: A Sociocultural Exploration. Londres: Sage.

Manzanos, C. y Balmaseda J. (2003). Situación de las mujeres en las cárceles del País Vasco. Vitoria-Gasteiz: Gobierno Vasco.

Martín Palomo, T., Miranda M.J. y Vega Solís, C. (eds.) (2005). Delitos y fronteras: Mujeres extranjeras en prisión. Madrid: Universidad Complutense de Madrid.

O.N.U. (2011). Reglas de las Naciones Unidas para el tratamiento de las reclusas y medidas no privativas de libertad para las mujeres delincuentes (Reglas de Bangkok). (2011). Recuperado de: http://www.penalreform.org/wpcontent/uploads/2013/06/United_Nations_Rules_for_the_Treatment_of_Women_Prisoners_and_Non-custodial_Measures_for_Women_Offenders_the_Bangkok_RulesSPANISH.pdf.

OSPDH. (2006). La cárcel en el entorno familiar. Barcelona: Ayuntamiento de Barcelona.

PRECA. (junio de 2011). Prevalencia de Trastornos Mentales En Centros Penitenciarios Españoles. Barcelona, Recuperado de: http://www.derechopenitenciario.com/comun/fichero.asp?id=2505.

Ribas, N., Ameda, E. y Bodelón, E. (2005). Rastreando lo invisible. Mujeres extranjeras en las cárceles. Barcelona: Anthropos.

Ribas, N. y Martínez J. (2003). Mujeres extranjeras en las cárceles españolas. Sociedad y Economía, $5,65-88$.

Ríos Martín, J. y Cabrera Cabrera, P. (1998). Mil voces presas. Madrid: Universidad Pontificia de Comillas.

Ríos Martín, J. y Cabrera Cabrera, P. (2002). Mirando el abismo. El régimen cerrado. Madrid: Universidad Pontificia de Comillas.

Salhaketa (2005). Observando una cárcel. Nanclares de Oca-Langraitz. Recuperado de: http://www.nodo50.org/tortura/varios/NANCLARES-2005.pdf.

San Martín, C. (2007). Discursos psicológicos difíciles de digerir o en torno a la psicopatologización generizada de los malestares. En: Estado de Wonderbra: Entretejiendo narraciones feministas sobre las violencias de género. Barcelona: Virus.

Sanfélix-Gimeno, G. et al. (2012). La prescripción farmacéutica en Atención Primaria. Informe SESPAS 2012. Gaceta Sanitaria, 26(S), 41-45.

Gañán, A. y Gordon, B. (2000). La mujer drogodependiente penada en los centros penitenciarios.

Smart, C. (1977). Women, crime and criminology: a feminist critique. Londres: Routledge and Kegan Paul. 
Worrall, A. (1990). Offending women: female lawbreakers and the criminal justice system. Londres y Nueva York: Routledge.

Valverde Molina, J. (1991). La cárcel y sus consecuencias. Intervención sobre la conducta desadaptada. Madrid: Popular.

Yagüe Olmos, C. (2007). Madres en prisión. Historia de las cárceles de mujeres a través de su vertiente maternal. Granada: Comares. 